\title{
Efectos diferenciales de los programas de educación en diabetes según los niveles de HbAlc y la presencia de complicaciones crónicas en el paciente tipo 1
}

\author{
Differential effects of diabetes education programs by levels \\ of HbA1c and the presence of chronic complications \\ in patients with type 1 diabetes
}

\author{
M. De los Santos-Roig ${ }^{1}$, M. Fernández-Alcántara ${ }^{1}$, T. Guardia-Archilla ${ }^{2}$, \\ S. Rodríguez-Morales ${ }^{2}$, A. Molina ${ }^{3}$, D. Casares ${ }^{3}$, I. Ruiz-González ${ }^{2}$
}

\section{RESUMEN}

Fundamento. Los programas de Educación Diabetológica (PED) mejoran el control metabólico pero son aplicados a pacientes de muy diversa índole. El objetivo es comprobar si un PED funciona de manera diferente según el perfil del paciente.

Material y Métodos. Participaron en el estudio 36 pacientes diagnosticados de diabetes tipo 1. Fueron segmentados en cuatro grupos según niveles de hemoglobina (rango de 7-13\%) y en dos, según la presencia o no de complicaciones. Todos cumplimentaron la Escala ECODI y la de Frecuencia de Auto-Cuidado.

Resultados. Se comprueba cómo tras el PED existe una disminución de la Hb1Ac y algunas áreas del auto-cuidado también mejoran, pero no hay cambios en dieta ni en el ejercicio.

Conclusiones. El PED parece funcionar mejor en los pacientes con peor control y con complicaciones, y se discute, por tanto, su papel preventivo. Se concluye sobre la necesidad de mejorar la intervención, ya que no hay cambios en aspectos relativos a los factores de riesgo cardiovascular, incluyendo estrategias psicológicas que motiven un cambio real en el estilo de vida.

Palabras clave: Diabetes tipo 1. Control metabólico. Programa educación intensiva. Habilidades. Auto-cuidado.

\begin{abstract}
Background. Diabetes Education Programs (DEP) that improve metabolic control are applied to a wide variety of patient types. The aim is to test whether DEPs work differently depending on the patient profile.

Materials and Methods. Thirty-six type 1 diabetics participated. They were divided into four groups according to their haemoglobin levels (range: $7-13 \%$ ) and into two groups according to the presence or absence of complications. The ECODI scale for assessing diabetes knowledge and the Frequency of Self-Care scale were completed by all patients.
\end{abstract}

Results. The results showed that HbAlc decreased after the DEP, with some areas of self-care also improving. There were no changes, however, to diet or exercise.

Conclusions. DEP appear to work better in patients with worse control and with complications, suggesting that they have a certain role to play in prevention. Their lack of impact on diet or exercise, would suggest that the DEPs require improvement to include psychological strategies that motivate lasting lifestyle changes.

Key words. Type 1 diabetes. Metabolic control. Intensive education program. Skill practice. Self-care.
1. Facultad de Psicología. Universidad de Granada (España)

2. Unidad de Gestión Clínica de Endocrinología y Nutrición. Hospital Universitario San Cecilio Hospital Universitario Virgen de las Nieves. Granada (España)

3. Unidad de Gestión Clínica de Endocrinología y Nutrición. Hospital Universitario San Cecilio. Granada (España)

Recepción: 10 de diciembre de 2013

Aceptación provisional: 21 de febrero de 2014

Aceptación definitiva: 4 de marzo de 2014

\section{Correspondencia:}

Macarena De los Santos Roig

Dpto. Metodología de las Ciencias del

Comportamiento. Facultad de Psicología

Universidad del Granada

18071. Granada (España)

E-mail: dlsantos@ugr.es

Proyecto de investigación financiado por la Consejería de Salud de la Junta de Andalucía. PI-00602010. 


\section{INTRODUCCIÓN}

Existe una amplia evidencia sobre la influencia positiva de los programas de educación diabetológica (PED) en el control metabólico y las complicaciones ${ }^{1,2}$. Aunque hay estudios que han abordado cuáles son los componentes y formato que deben incluir $^{3,4}$, lo cierto es que pocos de ellos se han centrado en estudiar el perfil del paciente que participa en ellos.

Este trabajo forma parte de un proyecto más amplio que comparó dos programas; uno de formato intensivo-práctico (4 sesiones consecutivas, que incluían la práctica específica de habilidades) y otro estándar (4 sesiones de carácter teórico, espaciadas en el tiempo). Ambos fueron implementados por enfermeras en dos hospitales de día de diabetes. Los resultados han señalado mejorías en los dos grupos, aunque más duraderas en el programa intensivo ${ }^{5}$.

El presente estudio se centra en conocer si el programa intensivo influye de manera diferente en los pacientes en función de sus características, ya que no todos se encuentran en la misma condición y muchas veces las complicaciones son percibidas como ajenas a uno mismo o muy lejanas en el tiempo. El objetivo es comprobar si todos los pacientes mejoran de igual forma tras el PED, o si, por el contrario, esta mejoría es significativamente mayor en aquellos pacientes con peor control metabólico y que presentan complicaciones.

\section{MATERIAL Y MÉTODOS}

Participaron un total de 36 pacientes con diabetes tipo 1 , todos con tratamiento bolo/basal y cuya media de edad fue de 36,64 años (Desviación típica 14,89). El $44,4 \%$ de ellos eran hombres y $11(31,4 \%)$ presentaban complicaciones crónicas; lo más frecuente fue la retinopatía $(15,2 \%)$ y la presencia de retinopatía junto a nefro o neuropatía (9\%).

Se realizó un estudio transversal prepost, con seguimiento al año, donde se registraron medidas sobre hemoglobina (HbA1c), conocimiento y frecuencia de autocuidado antes, después y a los 12 meses de la realización del PED.
Los instrumentos utilizados fueron la Escala de Conocimientos sobre la Diabetes -ECODI-, compuesta por 25 ítems con cuatro alternativas de respuesta. Sus índices de fiabilidad son de 0,87 y $0,86^{6}$. A estos ítems se les añadieron 4 más que evaluaban el conocimiento de tipo técnico, que fueron desarrollados para esta investigación y de cuyas puntuaciones, por el momento, no han podido obtenerse sus evidencias de fiabilidad y validez. Por este motivo, no se han tratado junto al resto de la escala. Para evaluar la frecuencia de autocuidado se utilizó una la Summary of Diabetes Self-Care Activities Measure (SDSCA) de Toobert y col que también cuenta con garantías psicométricas $^{7}$. El instrumento mide cuatro áreas de auto-cuidado (dieta, ejercicio, tratamiento y cuidado del pie).

Una vez dado el visto bueno del Comité Ético del hospital y obtenido el consentimiento informado de los pacientes, se realizó la primera evaluación antes de aplicar el PED. Al acabarlo, se volvió a evaluar al paciente y antes de darle el alta se estableció una cita para seis meses después.

El programa forma parte de las actividades del Hospital de Día (realizadas de 9 a 15 h.). Es impartido por la educadora y consta de 4 sesiones compuestas por dos partes: a) Parte teórica: de 2 horas de duración y b) Parte práctica: en este caso, de 3 horas cada una, con resolución de diversos casos prácticos (cálculo de carbohidratos, prevención y actuación ante la hipoglucemia, ajuste de dosis, actuación ante la actividad física, etc.) y supervisión-corrección de las habilidades aprendidas.

Se realizaron una serie de Ancova con el tiempo de evolución como co-variable. Las variables independientes se crearon segmentando a los participantes en grupos según el nivel de HbA1c inicial (G1HbA1c $<8 \%$, G2-HbA1c de $8 \%$ a $8,9 \%$ y G3HbAlc de 9 a $9,9 \%$ y G4-HbAlc $>9,9 \%$ ) y según la existencia de complicaciones crónicas (G1-Sí, G2-No). Los grupos según la HbA1c fueron conformados considerando los cuartiles, así como con el criterio basado en la experiencia clínica de las educadoras. Todos los análisis se realizaron con SPSS (v.20). 


\section{RESULTADOS}

La Hb1Ac baja en todo el grupo de 8,98 a $8,11 \%\left(\mathrm{~F}_{2,56}=3,47, \mathrm{p}=0,03\right)$. Al analizar los grupos según su control metabólico (1 al 4) se comprobó una mejora general de la
Hb1Ac, que fue significativa en los grupos 3 y 4 (Tabla 1, Fig. 1). En el caso de los grupos con y sin complicaciones, ocurre lo mismo; todos cambian, pero solo los que presentan complicaciones mejoran significativamente (Fig. 2).

Tabla 1. Resultados del cambio en el control metabólico de los grupos de estudio

\begin{tabular}{ccccccccc}
\hline $\begin{array}{c}\text { Hemoglobina } \\
\text { inicial }\end{array}$ & Pretest & Post-test & Seguimiento & $\boldsymbol{F}_{2, \mathbf{2 4}}$ & $\boldsymbol{p}$ & $\boldsymbol{n}$ & $\begin{array}{c}\text { Potencia del } \\
\text { contraste }\end{array}$ & $\begin{array}{c}\text { Tamaño } \\
\text { del efecto }\end{array}$ \\
\hline G1 & $7,35(0,54)$ & $7,21(0,69)$ & $7,24(0,81)$ & 0,08 & n.s. & 7 & - & - \\
G2 & $8,56(0,25)$ & $8,41(1,03)$ & $7,93(0,93)$ & 1,00 & n.s. & 8 & - & - \\
G3 & $9,44(0,29)$ & $8,24(0,95)$ & $8,17(1,27)$ & $\mathbf{8 , 8 7}$ & $\mathbf{0 , 0 0}$ & $\mathbf{1 0}$ & $\mathbf{0 , 9 5}$ & $\mathbf{0 , 4 2}$ \\
G4 & $10,98(0,7)$ & $9,48(0,98)$ & $9,94(1,90)$ & $\mathbf{6 , 7 5}$ & $\mathbf{0 , 0 0}$ & $\mathbf{5}$ & $\mathbf{0 , 8 8}$ & $\mathbf{0 , 3 8}$ \\
\hline Complicaciones & & & & $\boldsymbol{F}_{2,25}$ & & & & \\
\hline Sí & $9,47(0,85)$ & $8,53(0,57)$ & $8,26(0,98)$ & $\mathbf{5 , 4 1}$ & $\mathbf{0 , 0 1}$ & $\mathbf{8}$ & $\mathbf{0 , 3 3}$ & $\mathbf{0 , 2 5}$ \\
No & $8,76(8,76)$ & $8,13(11,31)$ & $8,11(1,63)$ & 2,63 & n.s. & 21 & & \\
\hline
\end{tabular}

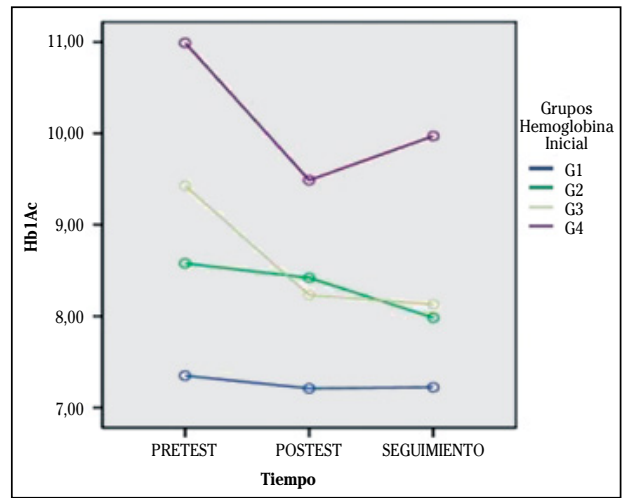

Figura 1. Resultados del cambio en Hb1Ac para los grupos de control metabólico inicial.

Con relación al conocimiento sobre la diabetes, el conocimiento teórico está cerca del máximo que permite la escala, y no se producen cambios. En la tabla 2 se muestra que hubo cambios significativos en el conocimiento técnico, en los grupos de hemoglobina intermedios (el G2 y el G3). Como se puede observar, las medias

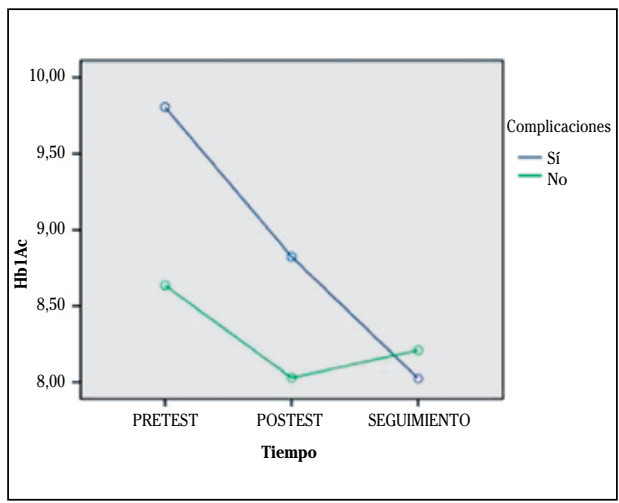

Figura 2. Resultados del cambio en la Hb1Ac para los grupos según complicaciones.

varían significativamente $\mathrm{y}$, por tanto, el Ancova, ofrece un resultado significativo para ambos grupos: $\mathrm{F}_{2,28}=4,47, \mathrm{p}=0,02$ y $\mathrm{F}_{2,28}=4,89, \mathrm{p}=0,01$. En los dos grupos según complicaciones-Si/No, los resultados también indican diferencias significativas en $\operatorname{los} \operatorname{dos}\left(\mathrm{F}_{2,29}=3,51, \mathrm{p}=0,04\right.$ y $\mathrm{F}_{2,29}=7,10$, $\mathrm{p}=0,00$, respectivamente). 
Tabla 2. Resultados del cambio en el conocimiento técnico y el auto-cuidado (tratamiento y cuidados del pie) de los grupos de estudio

\begin{tabular}{|c|c|c|c|c|c|c|c|c|}
\hline \multicolumn{9}{|c|}{ CONOCIMIENTO TÉCNICO } \\
\hline $\begin{array}{l}\text { Hemoglobina } \\
\text { Inicial }\end{array}$ & Pretest & Post-test & Seguimiento & $F_{2,28}$ & $\boldsymbol{p}$ & $n$ & $\begin{array}{l}\text { Potencia } \\
\text { contraste }\end{array}$ & $\begin{array}{c}\text { Tamaño } \\
\text { efecto }\end{array}$ \\
\hline G1 & $3,28(1,11)$ & $4,14(1,21)$ & $3,57(0,97)$ & 2,31 & n.s. & 7 & & \\
\hline G2 & $3,12(1,35)$ & $4,25(0,88)$ & $4,25(0,70)$ & 4,47 & $\mathbf{0 , 0 2}$ & 8 & 0,71 & 0,24 \\
\hline G3 & $2,83(1,40)$ & $4,00(0,95)$ & $3,83(1,26)$ & 4,89 & $\mathbf{0 , 0 1}$ & 12 & $\mathbf{0 , 7 6}$ & 0,26 \\
\hline G4 & $3,08(1,19)$ & $4,28(0,75)$ & $4,00(1,41)$ & 2,90 & n.s. & 7 & & \\
\hline Complicaciones & & & & $\mathbf{F}_{2,29}$ & & & & \\
\hline Sí & $3,00(1,26)$ & $4,36(1,02)$ & $3.81(1,32)$ & 3,51 & $\mathbf{0 , 0 4}$ & 11 & $\mathbf{0 , 6 0}$ & $\mathbf{0 , 1 9}$ \\
\hline No & $3,09(1,19)$ & $4,04(0,89)$ & $3,95(1,04)$ & 7,10 & $\mathbf{0 , 0 0}$ & 22 & $\mathbf{0 , 9 0}$ & $\mathbf{0 , 3 2}$ \\
\hline \multicolumn{9}{|c|}{ AUTOCUID. TRATAMIENTO } \\
\hline $\begin{array}{c}\text { Hemoglobina } \\
\text { Inicial }\end{array}$ & Pretest & Postest & Seguimiento & $F_{2,26}$ & $\boldsymbol{p}$ & $n$ & $\begin{array}{l}\text { Potencia } \\
\text { contraste }\end{array}$ & $\begin{array}{c}\text { Tamaño } \\
\text { efecto }\end{array}$ \\
\hline $\mathrm{G} 1$ & $12,00(3,65)$ & $13,86(0,37)$ & $12,00(2,30)$ & 2,82 & n.s. & 7 & & \\
\hline $\mathrm{G} 2$ & $9,71(4,57)$ & $11,71(2,75)$ & $13.28(0,95)$ & 1,99 & n.s. & 7 & & \\
\hline G3 & $10,92(3,61)$ & $12,00(2,08)$ & $11,07(3,14)$ & 0,90 & n.s. & 13 & & \\
\hline G4 & $10,20(3,42)$ & $12,80(1,30)$ & $10,80(4,38)$ & 3,52 & 0,04 & 5 & 0,60 & 0,21 \\
\hline Complicaciones & & & & $\mathbf{F}_{2,27}$ & & & & \\
\hline Sí & $11,30(3,16)$ & $12,30(1,33)$ & $11,40(3,06)$ & 0,11 & n.s. & 10 & & \\
\hline No & $10,81(3,89)$ & $12,71(2,19)$ & $11,80(2,94)$ & 6,72 & $\mathbf{0 , 0 0}$ & 21 & $\mathbf{0 , 8 8}$ & $\mathbf{0 , 3 3}$ \\
\hline \multicolumn{9}{|c|}{ AUTOCUID. CUIDADOS PIE } \\
\hline $\begin{array}{c}\text { Hemoglobina } \\
\text { Inicial }\end{array}$ & Pretest & Postest & Seguimiento & $F_{2,24}$ & $\boldsymbol{p}$ & $\boldsymbol{n}$ & $\begin{array}{l}\text { Potencia } \\
\text { contraste }\end{array}$ & $\begin{array}{c}\text { Tamaño } \\
\text { efecto }\end{array}$ \\
\hline G1 & $11,29(5,12)$ & $15,00(7,59)$ & $16,00(6,27)$ & 2,66 & n.s. & 7 & & \\
\hline $\mathrm{G} 2$ & $14,43(4,57)$ & $16,71(8,11)$ & $15,14(7,60)$ & 0,62 & n.s. & 7 & & \\
\hline G3 & $15,58(7,26)$ & $17,08(6,70)$ & $19,33(5,56)$ & 2,01 & n.s. & 12 & & \\
\hline G4 & $14,00(8,16)$ & $7,50(7,93)$ & $23,59(3,69)$ & 5,13 & $\mathbf{0 , 0 1}$ & 4 & $\mathbf{0 , 7 7}$ & $\mathbf{0 , 3 0}$ \\
\hline Complicaciones & & & & $\mathbf{F}_{2,25}$ & & & & \\
\hline Sí & $15,38(6,94)$ & $17,00(6,39)$ & $20,50(5,37)$ & 3,20 & $\mathbf{0 , 0 5}$ & 8 & 0,56 & $\mathbf{0 , 2 0}$ \\
\hline No & $13,76(6,26)$ & $14,48(8,35)$ & $17,38(6,79)$ & 1,44 & n.s. & 21 & & \\
\hline
\end{tabular}

El auto-cuidado no experimentó cambios en ninguno de los grupos ni en autocuidado total, ni en dieta, ni en ejercicio, por eso sólo se muestran los resultados parciales. Sin embargo, sí se encontraron diferencias significativas en el G4, el de peor control metabólico, concretamente en tratamiento y cuidado del pie. El Ancova para las puntuaciones en las dos subescalas ha resultado en $\mathrm{F}_{2,26}=3,52, \mathrm{p}=0,04$ y $_{2,24}=5,13, p=0,01$, respectivamente. Para aquellos con complicaciones, los cambios aparecieron cuidados del pie $\left(\mathrm{F}_{2,25}=3,20\right.$, $\mathrm{p}=0,05$ ), y en aquellos que no presentaban complicaciones se produce un cambio en el auto-cuidado del tratamiento $\left(\mathrm{F}_{2,27}=6,72\right.$, $\mathrm{p}=0,00$ ); como se observa, al finalizar el programa las puntuaciones medias aumentan, pero vuelven a disminuir a los 12 meses. 


\section{DISCUSIÓN}

El PED ayuda a que los pacientes mejoren; la hemoglobina disminuye significativamente y este dato debe valorarse positivamente desde un punto de vista clínico. La reducción general que se ha producido está cercana a un punto y pasar de 8 a $7 \%$ reduce entre el $17-21 \%$ y $24-33 \%$ el riesgo de retinopatía y de nefropatía, respectivamente $^{1-2}$. Además, reducir de 9 a $8 \%$ disminuye en un $35 \%$ los riesgos cardiovasculares $^{8}$. Sin embargo, teniendo en cuenta que los que mejoran sufren ya complicaciones con las consecuencias también económicas que ello comporta ${ }^{9}$, es muy importante valorar el efecto preventivo del PED. Los resultados de este estudio parecen indicar que los pacientes con mejor Hb1Ac (aunque mal controlados) y aquellos sin complicaciones, no se benefician tanto del programa. Probablemente la lejanía de las consecuencias de estar mal controlado, y la motivación para el cambio sea diferente en unos que en otros, etc. Por eso, sería muy útil introducir esos aspectos psicológicos en los PED.

Por otra parte, si se analizan los conocimientos, se puede observar cómo los pacientes conocen ya la enfermedad. Un área susceptible de mejora es el conocimiento de tipo técnico, el asociado a la técnica de administración de la insulina. En ese caso, se ha comprobado cómo el PED aún puede aportar algo si se incluyen esos aspectos prácticos y técnicos.

Finalmente, se ha visto cómo el autocuidado mejora en algunos casos, aunque no se alcanza el máximo posible. Las mayores puntuaciones aparecen en tratamiento. Sin embargo, no se producen cambios en dieta, ni en ejercicio, dos pilares fundamentales del estilo de vida saludable y altamente relacionados con la reducción de riesgo cardiovascular, muy presente en la $\mathrm{DM}^{10}$, ${ }^{11}$. Los cambios han sido mínimos y se han dado precisamente en el grupo más complicado, lo que lleva de nuevo a pensar en el escaso papel preventivo de los PED.

Como conclusión, puede decirse que, a pesar de la escasa muestra y las limitaciones del estudio, considerar las compli- caciones del paciente, así como su nivel de Hb1Ac (no es lo mismo un paciente con 8 que con $13 \%$ ) es fundamental para adaptar la educación a cada caso. Además, si se pretenden efectos más duraderos los programas deben centrarse en aumentar la motivación y promover el cambio en el estilo de vida. Cuando el conocimiento sobre la enfermedad ya se ha conseguido, el cambio de los hábitos (dieta y ejercicio) se puede lograr a través de estrategias psico-educativas por lo que se requiere de un equipo multidisciplinar que incluya profesionales de la psicología. Por último es importante tener en cuenta que la educación es un proceso continuo y que la distancia, el trabajo, los horarios, etc. no siempre ayudan a que los pacientes acudan a buscar solución del problema al equipo de salud, tomando decisiones que tal vez no sean las adecuadas. La incorporación de la nueva tecnología puede ayudar en parte. Esto, unido a lo anterior permitiría que los PED tuvieran mayor alcance y sus efectos fueran mucho más duraderos.

\section{BIBLIOGRAFÍA}

1. Shichiri M, Ohkubo Y, KishikaWa H, WaKe N. Long term results of the Kumamoto Study on optimal diabetes control in type 2 diabetic patients. Diab Care 2000; 23: Suppl. 2: B21-B2.

2. Diabetes Control and Complications Trial Research Group. The effect of intensive treatment of diabetes on the development and progression of long-term complications in insulin-dependent diabetes mellitus. N Engl J Med 1993; 329: 977-986.

3. Chodosh J, Morton SC, Mojica W, Maglione M, Suttorp MJ, HiLton L et al. Meta-analysis: chronic disease self- management programs for older adults. Ann Intern Med 2005; 143: 427-438.

4. Rickheim PL, Weaver TW, Flader JL, Kendall DM. Assessment of group versus individual diabetes education: a randomized study. Diab Care 2002; 25: 269-274.

5. Ruiz-González I, Fernández-Alcántara M, Guardia-Archilla T, Molina A, Casares D y De los SanTos-Roig M. Educación Diabetológica, ¿Qué podemos cambiar?: Efectos a largo plazo de un programa intensivo-práctico sobre el autocuidado del paciente con Diabetes tipo 1. Rev Paraninfo Digital, 2012; 16. 
6. Bueno JM, Marco MD, Leal A, Orozco D, Mira JJ. An evaluation study of a scale of diabetological education in primary care. Aten Primaria 1993; 11: 344-348.

7. Toobert DJ, Hampson SE, Glasgow RE. The summary of diabetes self-care activities measure: results from 7 studies and a revised scale. Diab Care 2000; 23: 943-950.

8. American Diabetes Association: Implications of the United Kingdom Prospective Diabetes Study (Position Statement). Diab Care 2002; 25 (Suppl. 1): S28-S32.
9. GonzÁlez M, Galíndez A. Estudio del coste de hospitalización de las complicaciones de la diabetes. An Sist Sanit Navar 1997; 20: 71-76.

10. Chillarón JJ, Flores-Le-Roux JA, Goday A, Benalges D, CARrera MJ, Puig J et al. Síndrome metabólico y diabetes mellitus tipo 1: prevalencia y factores relacionados. Rev Esp Cardiol 2010; 63: 423-429.

11. Álvarez-Sala LA, Suárez C, Mantilla T, Franch J, RUILOPE LM, BANEGAS JR et al. Estudio PREVENCAT: control del riesgo cardiovascular en atención primaria. Med Clin 2005; 124: 406-410. 